\title{
Organic Field Effect Transistors Based on Graphene and Hexagonal Boron Nitride Heterostructures
}

\section{Citation}

Kang, Seok Ju, Gwan-Hyoung Lee, Young-Jun Yu, Yue Zhao, Bumjung Kim, Kenji Watanabe, Takashi Taniguchi, James Hone, Philip Kim, and Colin Nuckolls. 2014. "Organic Field Effect Transistors Based on Graphene and Hexagonal Boron Nitride Heterostructures." Advanced Functional Materials 24 (32) (June 16): 5157-5163. doi:10.1002/adfm.201400348.

\section{Published Version}

doi:10.1002/adfm.201400348

\section{Permanent link}

http://nrs.harvard.edu/urn-3:HUL.InstRepos:34604211

\section{Terms of Use}

This article was downloaded from Harvard University's DASH repository, and is made available under the terms and conditions applicable to Open Access Policy Articles, as set forth at http:// nrs.harvard.edu/urn-3:HUL.InstRepos:dash.current.terms-of-use\#OAP

\section{Share Your Story}

The Harvard community has made this article openly available.

Please share how this access benefits you. Submit a story.

Accessibility 
DOI: 10.1002/ ((please add manuscript number))

Article type: Full paper

Title: Organic Field Effect Transistors Based on Graphene and Hexagonal Boron Nitride Heterostructures

Seok Ju Kang, Gwan-Hyoung Lee, Young-Jun Yu, Yue Zhao, Bumjung Kim, Kenji Watanabe, Takashi Taniguchi, James Hone, * Philip Kim, * and Colin Nuckolls*

Dr. S. J. Kang, Dr. B. Kim, Prof. C. Nuckolls

Department of Chemistry, Columbia University, New York, NY 10027, USA

E-mail: cn37@columbia.edu

Dr. G.-H. Lee, Prof. J. Hone

Department of Mechanical Engineering, Columbia University, New York, NY 10027, USAE-

E-mail: jh2228@columbia.edu

Dr. G.-H. Lee

Department of Materials Science and Engineering, Yonsei University, Seoul 120-749, Korea

Dr. Y.-J. Yu, Dr. Y. Zhao, Prof. P. Kim.

Department of Physics, Columbia University, New York, NY 10027, USA

E-mail:pk2015@columbia.edu

Dr. Y.-J. Yu

Creative Research Center for Graphene Electronics, Electronics and Telecommunications Research Institute(ETRI), Daejeon 305-700, Korea.

Dr. K. Watanabe, Dr. T. Taniguchi

National Institute for Materials Science, 1-1 Namiki, Tsukuba, 305-0044, Japan

* S.J.K., G.-H.L., Y.-J.Y. contributed equally to the work.

Keywords: Graphehe, hBN, OFET, Rubrene, CVD 
Enhancing the device performance of single crystal organic field effect transistors (OFETs) requires both optimized engineering of efficient injection of the carriers through the contact and improvement of the dielectric interface for reduction of traps and scattering centers. Since the accumulation and flow of charge carriers in operating organic FETs takes place in the first few layers of the semiconductor next to the dielectric, the mobility can be easily degraded by surface roughness, charge traps, and foreign molecules at the interface. Here, we demonstrate a novel structure for high performance rubrene OFETs employing graphene and hexagonal boron nitride ( $\mathrm{hBN}$ ) as the contacting electrodes and gate dielectric layer, respectively. These hetero-stacked OFETs were fabricated by lithography-free dry-transfer method that allows us to transfer graphene and hBN on top of an organic single crystal, forming atomically sharp interfaces and efficient charge carrier-injection electrodes without damage or contamination. The resulting heterostructured OFETs exhibit both high mobility and low operating gate voltage, opening up new strategy to make high performance OFETs and great potential for flexible electronics. 


\section{Introduction}

The performance of OFETs is often constrained by the carrier channel mobility limited by the dielectric-semiconductor interface. ${ }^{[1-5]}$ In particular, trap states, which can be induced by residual charge density in the dielectric or active layer, influence the threshold voltage, mobility, and hysteresis of transfer characteristics. Therefore, lots of efforts has been devoted to discover a thin dielectric that can maintain high interface quality combined with high gate efficiency to improve the performance of OFETs ${ }^{[1-2,6-9]}$ Previous studies have demonstrated that air-gap OFETs can preserve the high channel mobility of rubrene by eliminating the interface completely; ${ }^{[10]}$ however, these devices are not practical for most applications, due to difficulties in integration, and high operating voltage. Other promising dielectrics, such as polydimethylsiloxane (PDMS), CYTOP, and polymeric materials, also present fabrication challenges such as inter-diffusion of organic molecules into the PDMS, and residual organic solvent in polymeric dielectrics. ${ }^{[1-15]}$ Moreover, these materials are generally thick and possess a low dielectric constant, leading to high operating voltage. Recently, it was reported hexagonal boron nitride (hBN), an insulating isomorph of graphene, is a highly optimal substrate and dielectric for graphene FETs due to its atomically flat surface and the absence of trapped interfacial charge. ${ }^{[16-17]}$ For this reason, hBN can be similarly utilized as a dielectric for high performance and low-power consumption OFETs. An additional challenge for OFETs device fabrication is the need for a nondestructive lithography method because conventional solvent-based lithographic processing can cause significant damage to organic materials. ${ }^{[18]}$

In this study, we demonstrate a novel structure of high performance OFETs by stacking rubrene single crystal, graphene, and $\mathrm{hBN}$, which were employed as channel, electrode, and dielectric, respectively. The stacked OFETs were fabricated by lithography-free PDMS 
transfer method, which allowed us to form a atomically-flat channel interface between rubrene and $\mathrm{hBN}$, and efficient charge-injection graphene electrodes without contamination.

\section{Result and discussion}

Rubrene single crystals were grown in a quartz tube by physical vapor transport (PVT) and subsequently transferred onto the silicon substrate with 300 nm-thick $\mathrm{SiO}_{2}$ capping layer, using a static gun. ${ }^{[18-19]}$ Figure 1a shows a representative rubrene single crystal. Rod-like rubrene single crystals with thickness of $100 \sim 200 \mathrm{~nm}$ and width of $10 \sim 50 \mu \mathrm{m}$ were selected for fabrication of OFET devices. The longitudinal direction of rubrene crystal corresponds to the $b$-direction, which is confirmed by selected area electron diffraction (SAED) (see below for details). We used rod-like rubrene crystals for OFET devices so that all the devices used in this work have $b$-axis of rubrene as a channel direction, which has been known as the highest mobility direction of rubrene. ${ }^{[18]}$ The hBN flakes were prepared with mechanical exfoliation method on a PDMS stamp which has a step-free and clean surface (Figure 1b). We further performed atomic force microscopy (AFM) to investigate the surface morphology of rubrene and hBN. Based on the normalized histograms of surface roughness analyzed from the AFM images, the surfaces of rubrene and $\mathrm{hBN}$ are more than three times smoother than $\mathrm{SiO}_{2}$ substrates (Figure 1c). It should be noted that the relatively thick rubrene crystals ( $>200$ $\mathrm{nm})$ have a number of molecular step-edges on the surface meanwhile the thin ones $(<200$ $\mathrm{nm}$ ) do not have ${ }^{[20-21]}$ (see Supporting Information, Figure S1). Thus, we judiciously selected rubrene crystals thinner than $200 \mathrm{~nm}$ for our devices. The van der Waals interaction between two ultra-flat hBN and rubrene crystals can lead to homogenously sharp interface after hetero-stacking process. ${ }^{[16,22]}$

The atomic scale quality of interface and crystal orientation of rubrene / hBN stack was also investigated using bright-field transmission electron microscopy (BF-TEM) and SAED 
pattern, respectively. We prepared a stack of rubrene and hBN on an amorphous carbon coated TEM grid using PDMS transfer technique. The BF-TEM image of the stack in Figure $1 \mathrm{~d}$ indicates that there are no trapped bubbles or molecular clusters. The electron diffraction of the stacked region (Figure 1e) exhibits the overlapped rectangular electron diffraction of rubrene $(h k 0)$ and hexagonal electron diffraction of $\mathrm{hBN}(0001)$, confirming that rubrene and $\mathrm{hBN}$ are single crystals and longitudinal direction of rubrene is $b$-axis. ${ }^{[19]}$

Figure 2a shows a schematic of the device fabrication process. Single-layer graphene films (CVD-SLG) were grown by chemical vapor deposition (CVD) on copper foil as reported elasewhere. ${ }^{[23]}$ CVD-SLG electrodes were transferred onto the top of the rubrene single crystal on the wafer substrate by using a patterned PDMS mold, as we have previously described. ${ }^{[19]}$ Note that, during this transfer process, the channel surface of the rubrene single crystal was untouched. Then, a hBN flake on PDMS stamp was transferred onto the channel area of the rubrene single crystal using a three-axis micro-manipulator. ${ }^{[16-17]}$ Following a similar method, few-layer graphene (FLG) was stacked onto the hBN flake to serve as a topgate electrode. Figure $2 \mathrm{~b}$ shows detailed process for aligning and transfering target flakes using three-axis micro-manipulator. The final device was heated in vacuum at $80^{\circ} \mathrm{C}$ for $24 \mathrm{~h}$ to ensure full contact between layers. The optical micrographs in Figure 3a-c show the device at each fabrication step. The schematic structure of final devices is shown in Figure 3d. In this structure, a gate voltage can be applied with either the bottom-gate $p$-doped Si or the topgate FLG. The AFM image of Figure 3e shows the morphology of the representative device of Figure $3 \mathrm{~d}$ before transfer of top-gate FLG layer. As shown in line profile of Figure 3f, the thicknesses of the rubrene crystal and $\mathrm{hBN}$ flake are $170 \mathrm{~nm}$ and $30 \mathrm{~nm}$, respectively. As already confirmed by BF-TEM (Figure 1d), the AFM image also shows no bubbles or residue in the channel interface between the rubrene and hBN layers. This stacking method obviates the need for conventional lithographic processing, which involves solvents. Therefore, we 
can easily fabricate devices with high reproducibilty, minimizing any damage during the process.

The OFETs can be electrically tested by directly contacting the source, drain, and gate electrodes using micromanipulator probes. In particular, the device structure of Figure $3 \mathrm{~d}$ allows us to characterize either top-gate or bottom-gate transistor geometry for direct comparison of device performance on the same rubrene single crystal. Figure $4 \mathrm{a}$ and b show transfer $\left(\mathrm{I}_{\mathrm{DS}}-\mathrm{V}_{\mathrm{G}}\right)$ and output $\left(\mathrm{I}_{\mathrm{DS}}-\mathrm{V}_{\mathrm{DS}}\right)$ characteristics in the bottom gate-top contact (BGTC) configuration measured under ambient condition. The $p$-type current modulation with operating voltage of $60 \mathrm{~V}$ and on / off ratio of approximately $10^{4}$ was observed. The field effect mobility of the OFET was calculated from MOSFET standard model in the saturated regime. The estimated mobility of this $\mathrm{BG}-\mathrm{TC}$ device was less than $1 \mathrm{~cm}^{2} / \mathrm{Vs}$ using the gate capacitance $\left(11.5 \mathrm{nF} / \mathrm{cm}^{2}\right)$ of $300 \mathrm{~nm}$ thick $\mathrm{SiO}_{2}$. This low mobility value was typical for our rubrene OFETs using $\mathrm{SiO}_{2}$ as gate dielectric. Moreover, for most of BG-TC devices, large shift of threshold voltage was observed, indicating that interface between rubrene single crystal and $\mathrm{SiO}_{2}$ has a large density of trap states. ${ }^{[24]}$

In contrast, greatly improved FET characteristics were measured for the device in the top gate-top contact (TG-TC) configuration (Figure 4c and d) using similarly grown rubrene

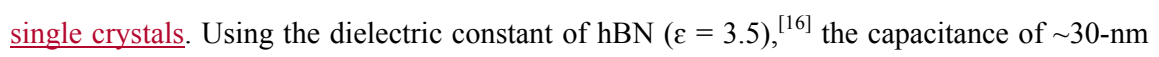
thick hBN was estimated to be $\sim 100 \mathrm{nF} / \mathrm{cm}^{2}$, which is an order of magnitude higher than that of thick $\mathrm{SiO}_{2}$ used in the BG-TC device. This allows the TG-TC device to operate with much lower gate voltage. For this TG-TC configuration, the On / Off ratio of $\sim 10^{7}$ was observed at low gate voltage of $5 \mathrm{~V}$. The field-effect mobilities of TG-TC OFETs extracted from the saturation regime are $10 \sim 15 \mathrm{~cm}^{2} / \mathrm{Vs}$ (from five devices), which are carrier densityindependent. Quantitatively, we found taht $I_{d}^{1 / 2} \sim V_{g}$ in the saturation regime, indicating uniform charge carriers and mobility along the channel. ${ }^{[25]}$ The output curves in Figure $4 d$

\begin{tabular}{|l|}
\hline Formatted: Font:Italic \\
\hline Formatted: Font:Italic, Subscript \\
\hline Formatted: Superscript \\
\hline Formatted: Font:Italic \\
Formatted: Font:Italic, Subscript \\
Deleted: Since linear increase of square-root of drain current in \\
saturation regime means \\
\hline Deleted: that \\
Deleted: are generated uniformly along the channel, \\
Deleted: is constant \\
\hline
\end{tabular}

Deleted: same Deleted: is constant 
show p-type characteristics with current saturation beyond $\mathrm{V}_{\mathrm{DS}}$ of $-5 \mathrm{~V}$ with stepped gate field modulation. As shown in the inset of Fig. 4d. the linear increase in drain current at small $\mathrm{V}_{\mathrm{DS}}$ indicates that a junction of CVD-SLG and rubrene forms an Ohmic contact. It can be explained in terms of well-alignment between Fermi level of highly $p$-doped CVD-SLG and HOMO level of rubrene ${ }^{[19,26-27]}$ This result suggest that highly $p$-doped CVD-SLG (increasing its work function) leads to closely align its Fermi level to HOMO level of rubrene Deleted: Namely (see Supporting Information, Figure S2). In contrast, the slow turn-on seen in the zoom-in output curves in the inset of Figure $4 \mathrm{~b}$ indicates that the BG-TC device has higher contact resistance due to the vertical separation between the top contacts and the bottom conducting channel. ${ }^{[28]}$ Furthermore, the TG-TC devices provides no hysteresis in the transfer curves which can be explained by the charge trap-free interface, i.e. clean $\mathrm{hBN} /$ rubrene interface (see Supporting Information, Figure S3). When the TG-TC device was measured in a linear regime (see Supporting Information, Figure S4a), field effect mobility increased as a function of gate voltage above threshold voltage and highest value was maintained at higher gate voltage region. This result indicates that channel / dielectric interface is clean and charge carriers are uniformly generated even in a linear regime of high carrier density. ${ }^{[29]}$ Whereas, the BG-TC configuration leads to the highest value near threshold voltage and decrease to very low value due to the high density of scattering charge at the interface (see Supporting Information, Figure S4b). We also found that the device performance of TG-TC configuration is more stable under ambient condition over several months, compared to other device configurations. This is probably because intimate contact of rubrene and hBN prevent any degradation of channel interface from penentration of water or gases.

To verify that the observed high performance is due to the novel device configuration, OFETs with various device structures were fabricated by using different dielectrics and electrode materials. (see Supporting Information, Figure. S5 and S6). Note that at least three 
devices for each different device configurations were fabricated and measured. Similar to previous work, rubrene single crystal FETs with air-gap dielectric and Au electrodes (Figire S5a) showed high mobility of $13 \sim 20 \mathrm{~cm}^{2} / \mathrm{Vs}$ as reported by other groups. ${ }^{[10,30-31]}$ When different electrodes such as evaporated thin gold (20 nm, Figure S5b) or exfoliated FLG (Figure S5c) were used in bottom gate-bottom contact (BG-BC) device configuration with hBN dielectric, the mobility $\left(3 \sim 4 \mathrm{~cm}^{2} / \mathrm{Vs}\right)$ was lower than that of the devices with CVDSLG electrodes (see Supporting Information, Table. S1). The low mobility of these devices is attributed to the residues of resist employed for e-beam lithography and bending of rubrene crystal at the edges of relatively thick electrode and hBN (device in Figure S5b). Using CVDSLG and hBN as contacts and dielectric in a BG-BC device (Figure S5d) resulted in high mobility similar to that of TG-TC devices, but with high operating voltage.

\section{Conclusion}

In conclusion, we demonstrate fabrication of heterostructured OFETs consisting of rubrene single crystal, graphene, and hBN, which serve as channel, source-drain electrode and dielectric. By employing a PDMS transfer method along with hBN, atomically flat and trapfree channel interface can be produced without damaging the organic materials or contaminating the interfaces. Charge carrier injection was improved by using highly-doped CVD-SLG electrodes. This straightforward and lithography-free fabrication method and novel device structure of TG-TC enable us to produce high performance OFETs with high field effect mobility, high on / off ratio, and low operating voltage by incorporating inorganic 2D materials into OFETs. This scheme opens up new strategy to make high performance OFETs by stacking 2D materials with great potential for flexible electronics.

\section{Experimental Section}

Growth of materials 
Rubrene single crystals were grown by physical vapor transport (PVT) as reported. ${ }^{[19]} 2 \mathrm{mg}$ of sublimed rubrene powder $(>99.5 \%$ ) was placed in a hot zone of the furnace. The substrate was placed in the crystallization zone $\left(280^{\circ} \mathrm{C}\right) .50 \mathrm{sccm}$ of a argon $(99.999 \%)$ was used as a carrier gas. After 5 minutes of pre-purging, the system was heated to $330{ }^{\circ} \mathrm{C}$. The reaction was performed for $2 \mathrm{~min}$ and the system was then cooled down to the room temperature. Single-layer graphene film was grown by CVD on $25 \mu$-thick copper foils (Alfa Aesar, $99.8 \%) .{ }^{[23]}$ The copper foil was heated to $1000{ }^{\circ} \mathrm{C}$ in a hydrogen flow of $2 \mathrm{sccm}$ at a pressure of 50 mTorr. After annealing for $60 \mathrm{~min}$, graphene was grown by introducing methane gas flow of $35 \mathrm{sccm}$ while maintaining hydrogen flow. After growth at $300 \mathrm{mT}$ orr and $1000{ }^{\circ} \mathrm{C}$ for $30 \mathrm{~min}$, the sample was rapidly cooled to ambient temperature under a flow of methane and hydrogen. After growth, a patterned PDMS mold was attached onto the graphene, and the copper foil was etched by ammonium persulfate for $2 \mathrm{~h}$.

\section{Device fabrication}

Rod-like rubrene single crystals on $\mathrm{SiO}_{2} / \mathrm{Si}$ substrate were selected for fabrication of OFET devices. CVD-SLG electrodes was transferred onto the top of rubrene single crystal with alignment in optical manipulator. A hBN flake was then transferred by using an unpatterned PDMS stamp. FLG was stacked onto the hBN flake by the same process to serve as a topgate electrode. The final device was heated in vacuum at $80^{\circ} \mathrm{C}$ for $24 \mathrm{~h}$ to ensure full contact between layers.

\section{Characterization of materials}

AFM (XE-100, Park Systems) and TEM (JEOL, JEM-100CX) were used to characterize the interface between layers and crystallography. hBN flake was deposited onto holy carbon grid and rubrene single crystal was transferred onto it using PDMS transfer technique. TEM imaging and diffraction were conducted with low operation voltage of $80 \mathrm{kV}$ to avoid any apparent damage. 


\section{Electrical characterization of devices}

Electrical properties of fabricated devices were measured with a semiconductor parameter analyzer (Agilent, 4155C) in air and at room temperature. The graphene electrodes were directly contacted by electrical probes in a micro-manipulator probe station.

\section{Supporting Information}

Supporting Information is available from the Wiley Online Library or from the author.

\section{Acknowledgements}

This research was primarily supported by the Department of Energy through the EFRC program (Grant DE-SC0001085). This material is based upon work supported by the National Science Foundation under Grant No. DMR-1122594 and by the Center on Functional Engineered Nano Architectonics (FENA) under award number 2009-NT-2048, subaward: UCLA 0160 S MB 959. P.K. and C.N. also acknowledges that the measurement characterization is supported by the FAME through STARNET. G.H.L. acknowledges support from Samsung-SKKU graphene center (SSGC).

Received: ((will be filled in by the editorial staff))

Revised: ((will be filled in by the editorial staff))

Published online: ((will be filled in by the editorial staff)) 


\section{References}

[1] O. D. Jurchescu, M. Popinciuc, B. J. van Wees, T. T. M. Palstra, Advanced Materials 2007, 19, 688

[2] J. Takeya, M. Yamagishi, Y. Tominari, R. Hirahara, Y. Nakazawa, T. Nishikawa, T. Kawase, T. Shimoda, S. Ogawa, Appl Phys Lett 2007, 90, 102120.

[3] Y. Don Park, J. A. Lim, H. S. Lee, K. Cho, Materials Today 2007, 10, 46.

[4] J. Veres, S. Ogier, G. Lloyd, D. de Leeuw, Chemistry of Materials 2004, 16, 4543.

[5] C.-a. Di, Y. Liu, G. Yu, D. Zhu, Accounts of Chemical Research 2009, 42, 1573.

[6] T. Takahashi, T. Takenobu, J. Takeya, Y. Iwasa, Appl Phys Lett 2006, 88, 033505.

[7] N. Benson, A. Gassmann, E. Mankel, T. Mayer, C. Melzer, R. Schmechel, H. von Seggern, J Appl Phys 2008, 104, 054505.

[8] A. von Muhlenen, M. Castellani, M. Schaer, L. Zuppiroli, Phys Status Solidi B 2008 245,1170 .

[9] J. Veres, S. D. Ogier, S. W. Leeming, D. C. Cupertino, S. Mohialdin Khaffaf, Advanced Functional Materials 2003, 13, 199.

[10] E. Menard, V. Podzorov, S. H. Hur, A. Gaur, M. E. Gershenson, J. A. Rogers, Advanced Materials 2004, 16, 2097.

[11] C. Reese, Z. Bao, Advanced Materials 2007, 19, 4535.

[12] X. Cheng, M. Caironi, Y.-Y. Noh, J. Wang, C. Newman, H. Yan, A. Facchetti, H. Sirringhaus, Chemistry of Materials 2010, 22, 1559.

[13] A. Facchetti, M. H. Yoon, T. J. Marks, Advanced Materials 2005, 17, 1705.

[14] I. Bae, S. J. Kang, Y. J. Shin, Y. J. Park, R. H. Kim, F. Mathevet, C. Park, Advanced Materials 2011, 23, 3398.

[15] F.-Y. Yang, K.-J. Chang, M.-Y. Hsu, C.-C. Liu, Journal of Materials Chemistry 2008, $18,5927$.

[16] C. R. Dean, A. F. Young, I. Meric, C. Lee, L. Wang, S. Sorgenfrei, K. Watanabe, T. Taniguchi, P. Kim, K. L. Shepard, J. Hone, Nat Nanotechnol 2010, 5, 722.

[17] C. R. Dean, A. F. Young, P. Cadden-Zimansky, L. Wang, H. Ren, K. Watanabe, T. Taniguchi, P. Kim, J. Hone, K. L. Shepard, Nat Phys 2011, 7, 693.

[18] V. C. Sundar, J. Zaumseil, V. Podzorov, E. Menard, R. L. Willett, T. Someya, M. E. Gershenson, J. A. Rogers, Science 2004, 303, 1644.

[19] S. J. Kang, B. Kim, K. S. Kim, Y. Zhao, Z. Y. Chen, G. H. Lee, J. Hone, P. Kim, C. Nuckolls, Advanced Materials 2011, 23, 3531 .

[20] A. L. Briseno, R. J. Tseng, M. M. Ling, E. H. L. Falcao, Y. Yang, F. Wudl, Z. N. Bao, Advanced Materials 2006, 18, 2320.

[21] T. Minato, H. Aoki, H. Fukidome, T. Wagner, K. Itaya, Appl Phys Lett 2009, 95.

[22] S. J. Haigh, A. Gholinia, R. Jalil, S. Romani, L. Britnell, D. C. Elias, K. S. Novoselov, L. A. Ponomarenko, A. K. Geim, R. Gorbachev, Nat Mater 2012, 11, 764.

[23] X. S. Li, W. W. Cai, J. H. An, S. Kim, J. Nah, D. X. Yang, R. Piner, A. Velamakanni, I. Jung, E. Tutuc, S. K. Banerjee, L. Colombo, R. S. Ruoff, Science 2009, 324, 1312.

[24] K. P. Pernstich, S. Haas, D. Oberhoff, C. Goldmann, D. J. Gundlach, B. Batlogg, A. N. Rashid, G. Schitter, J Appl Phys 2004, 96, 6431.

[25] D. Braga, G. Horowitz, Advanced Materials 2009, 21, 1473.

[26] W. H. Lee, J. Park, S. H. Sim, S. B. Jo, K. S. Kim, B. H. Hong, K. Cho, Advanced Materials 2011, 23, 1752.

[27] S. B. Jo, J. Park, W. H. Lee, K. Cho, B. H. Hong, Solid State Commun 2012, 152, 1350 .

[28] P. V. Necliudov, M. S. Shur, D. J. Gundlach, T. N. Jackson, J Appl Phys 2000, 88, 6594. 
[29] A. F. Stassen, R. W. I. de Boer, N. N. Iosad, A. F. Morpurgo, Appl Phys Lett 2004, 85, 3899.

[30] V. Podzorov, E. Menard, A. Borissov, V. Kiryukhin, J. A. Rogers, M. E. Gershenson, Phys Rev Lett 2004, 93, 086602.

[31] I. N. Hulea, S. Fratini, H. Xie, C. L. Mulder, N. N. Iossad, G. Rastelli, S. Ciuchi, A. F. Morpurgo, Nat Mater 2006, 5, 982. 
a
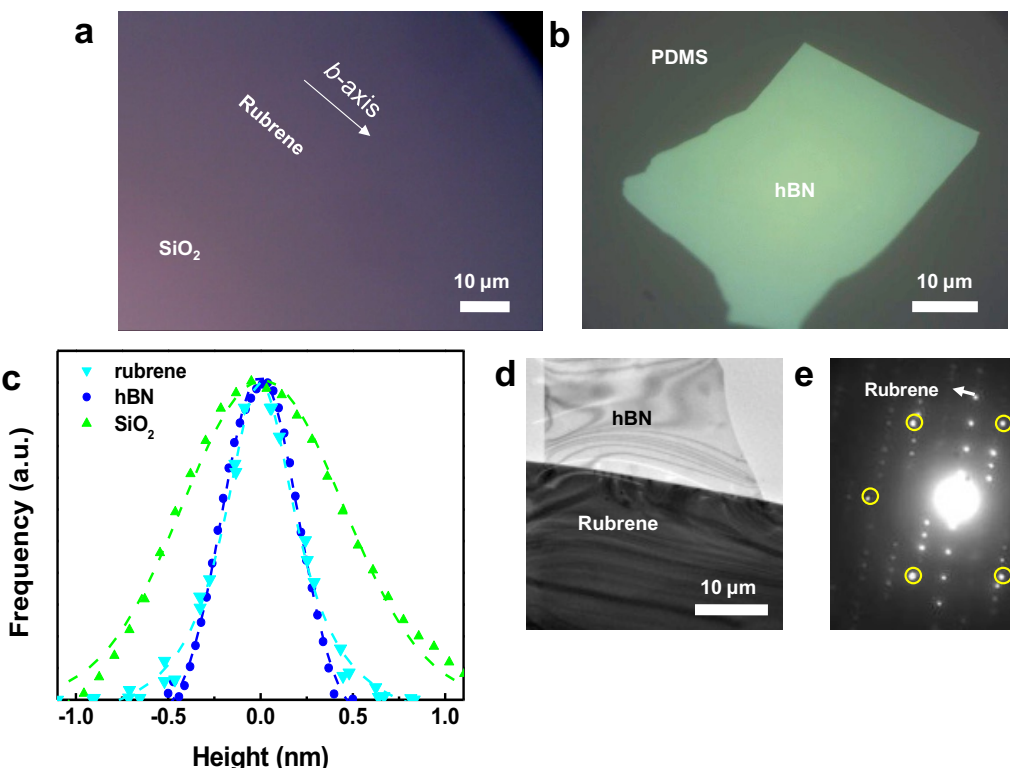

d

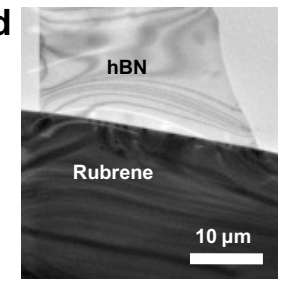

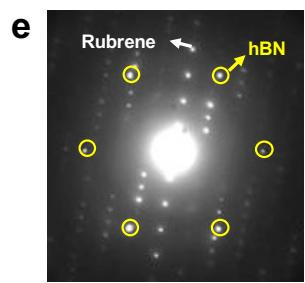

Height (nm)

Figure 1. Optical micrographs of (a) rubrene single crystal on $\mathrm{SiO}_{2} / \mathrm{Si}$ substrate and (b) hBN flake on PDMS stamp. The arrow in (a) indicates $b$-axis of the rubrene crystal. (c) Histograms of height distribution measured by AFM for rubrene, hBN, and $\mathrm{SiO}_{2}$. The AFM results indicate that the surface of rubrene and $\mathrm{hBN}$ is ultra-flat compared to $\mathrm{SiO}_{2}$. (d) BFTEM image and (e) SAED pattern of the stacked layers of rubrene / hBN. The yellow-circled electron diffraction spots show a hexagonal symmetry of hBN single crystal and the other spots are from rubrene single crystal. 
a
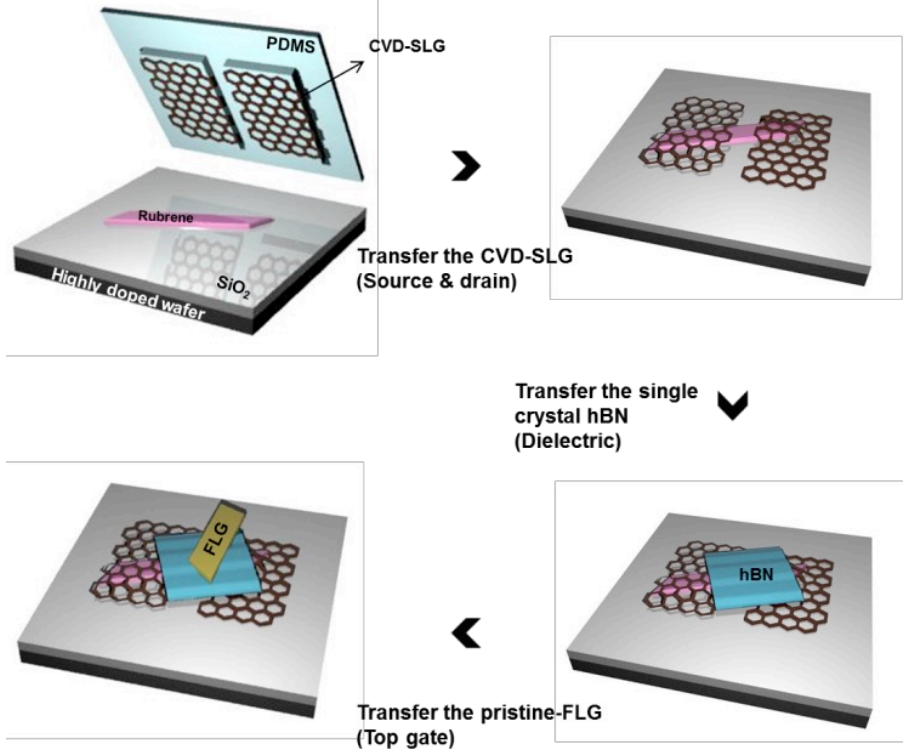

b

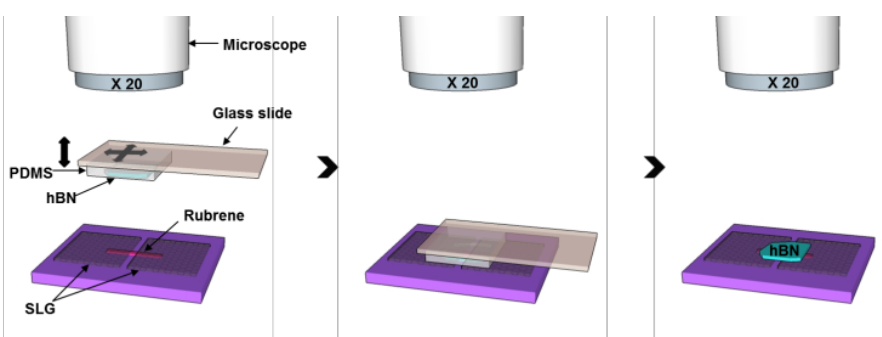

Figure 2. (a) schematic device fabrication process. Rubrene single crystal FETs were fabricated by stacking CVD-SLG, hBN, and pristine-FLG as electrodes, dielectric, and topgate electrode, respectively. (b) Schematic for alignment step in transfer process. The hBN flake is transferred onto channel region between two CVD-SLG electrodes by using threeaxis micro-manipulator. 
a

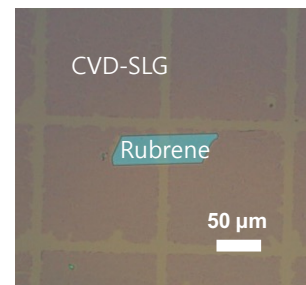

d

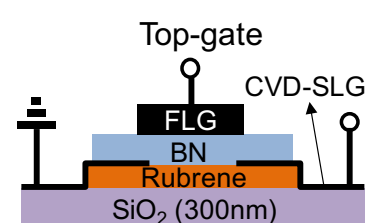

Highly doped Si wafer

Bottom-gate
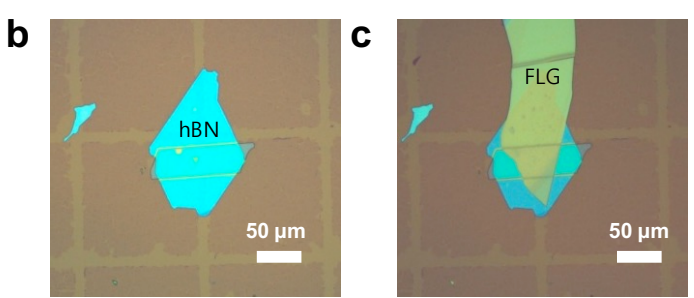

e
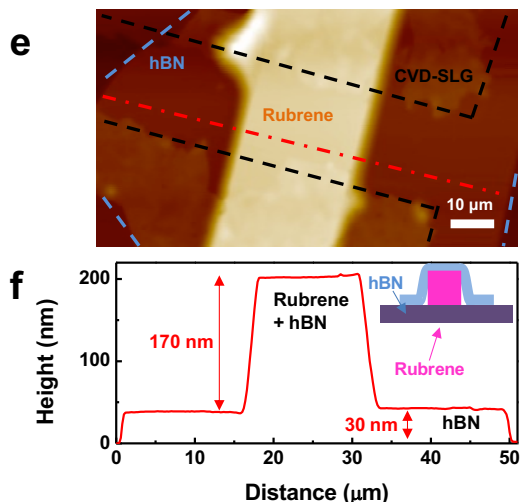

Figure 3. Optical micrographs of (a) Rubrene single crystal covered by CVD-SLG electrodes, (b) Channel area covered by hBN flake, and (c) Transfer of top-gate pristine-FLG electrode. (d) Schematic circuit diagram of the final device structure. (e) AFM image of the stacked layers of rubrene / CVD-SLG / hBN on $\mathrm{SiO}_{2} / \mathrm{Si}$ substrate. The dashed lines indicate the border of each layer. (f) Line profile measured from red dash-dot line of (e). The inset of (f) shows the cross-section structure of the $30 \mathrm{~nm}$ thick hBN on $170 \mathrm{~nm}$ thick rubrene crystal. 
a
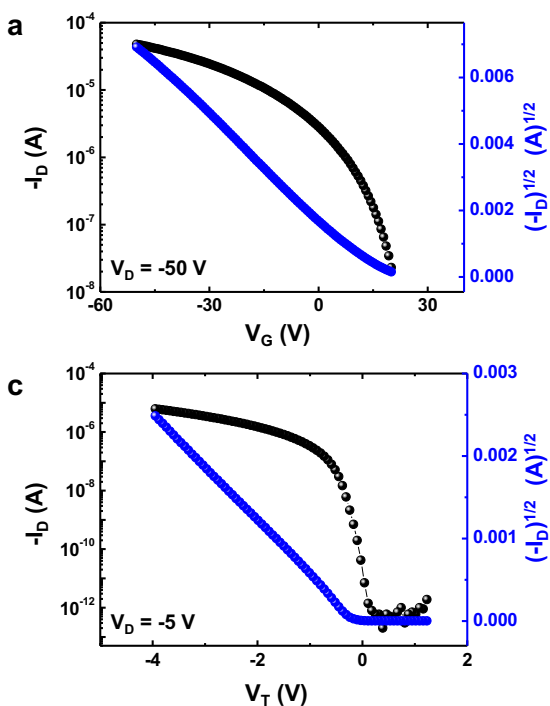

b
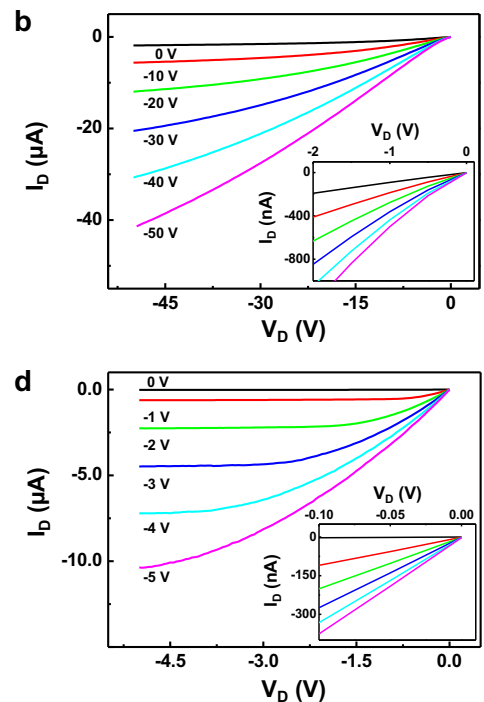

Figure 4. (a) Transfer curve and (b) Output curves of BG-TC configuration in the fabricated rubrene FET. (c) Transfer curve and (d) Output curves of TG-TC configuration of the same device. The insets of (b) and (d) show the zoom-in output curves at low bias region of the main panel. 
Copyright WILEY-VCH Verlag GmbH \& Co. KGaA, 69469 Weinheim, Germany, 2013.

\section{Supporting Information}

Title: Organic Field Effect Transistors Based on Graphene and Hexagonal Boron Nitride Heterostructures

Seok Ju Kang, Gwan-Hyoung Lee, Young-Jun Yu, Yue Zhao, Bumjung Kim, Kenji Watanabe, Takashi Taniguchi, James Hone, * Philip Kim, * and Colin Nuckolls* 


\section{Surface roughness of rubrene crystals}
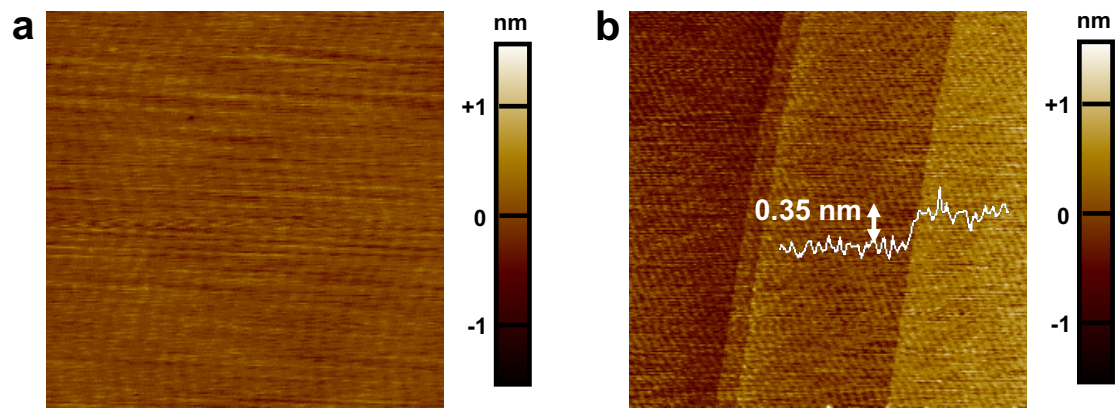

Figure S1. AFM images $(2.5 \mu \mathrm{m} \times 2.5 \mu \mathrm{m})$ of (a) $154 \mathrm{~nm}$ and (b) $396 \mathrm{~nm}$-thick rubrene single crystals. Thin rubrene single crystal has an ultra-flat and molecular step-free surface.

On the other hand, thicker one has a few steps of $0.35 \mathrm{~nm}$ in height. Note that the surface between step edges is also flat. 


\section{WILEY-VCH}

2. Electrical properties of CVD-SLG.
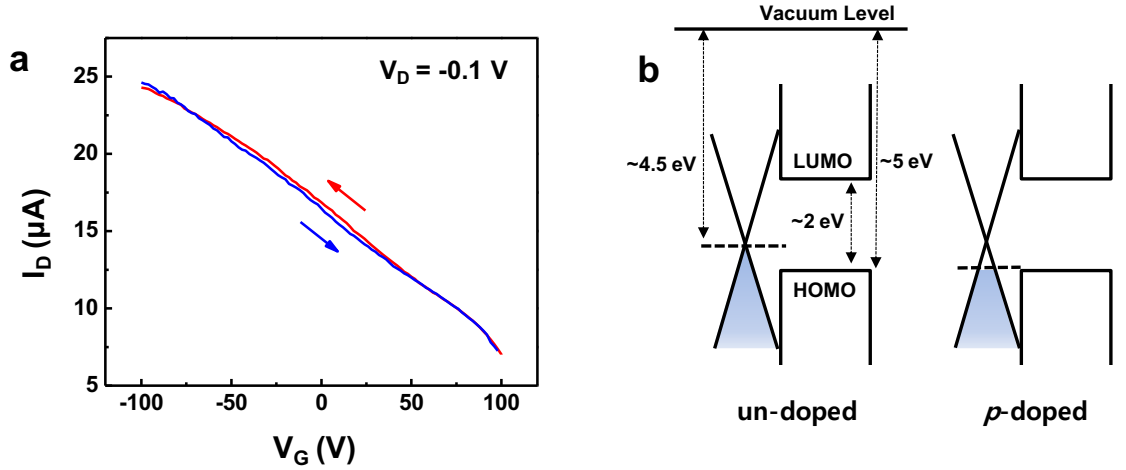

Figure S2. (a) Carrier transfer curve of CVD-SLG FET, which is used as S / D electrodes, shows a positive shift of charge neutral point $(>100 \mathrm{~V})$, indicating that CVD-SLG is highly $p$-doped. (b) Schematic band diagrams of rubrene-graphene junction for un-doped and p-doped graphene. When graphene is un-doped, Fermi level of graphene is away from HOMO level of rubrene: meanwhile the Fermi level in highly $p$-doped CVD-SLG is much lower than charge neutral point (increase of graphene's work function) and is initially leaned toward HOMO level of rubrene, leading to Ohmic contact. 


\section{WILEY-VCH}

\section{Transistor operation from BG-TC and TG-TC}
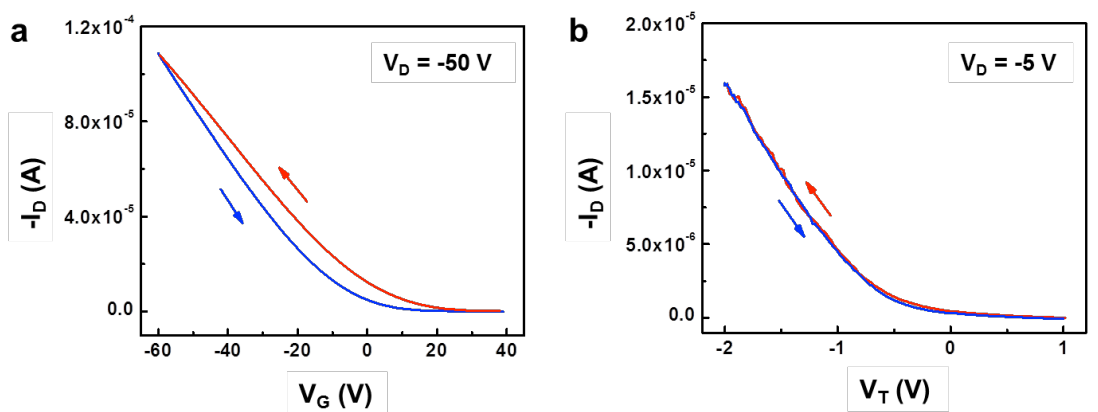

Figure S3. Carrier transfer curves of (a) BG-TC (rubrene / $\mathrm{SiO}_{2}$ interface channel) and (b)

TG-TC (hBN / rubrene interface channel) devices. The arrows indicate the directions of gate voltage sweep. The TG-TC device shows no hysteresis because of ultraclean and charge trapfree interface of $\mathrm{hBN} /$ rubrene. 


\section{WILEY-VCH}
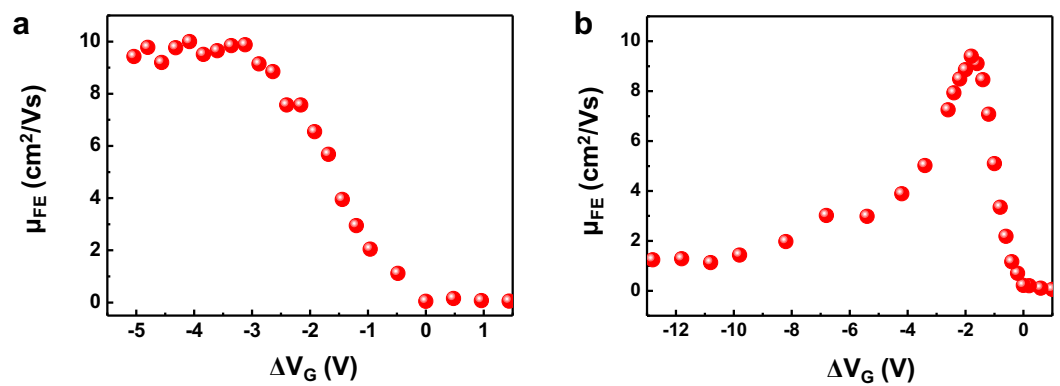

Figure S4. Field effect mobilities as a function of gate voltage $\left(\Delta \mathrm{V}_{\mathrm{G}}=\mathrm{V}_{\mathrm{G}}-\mathrm{V}_{\mathrm{Th}}\right)$ of (a) rubrene OFET on $\mathrm{hBN}$ and (b) on $\mathrm{SiO}_{2} / \mathrm{Si}$ substrate. The mobilities were obtained from measurements in a linear regime. 


\section{WILEY-VCH}

\section{Device structures and fabrication}

The devices with various structures were fabricated as shown in Figure S5 and S6. For air-gap device, the Si substrate with 300 nm-thick $\mathrm{SiO}_{2}$ was patterned through e-beam lithography and deposition of gold electrode. Using gold electrode as a mask, $\mathrm{SiO}_{2}$ capping layer was etched by reactive ion etching (RIE). Then, rubrene single crystal was transferred by PDMS transfer technique. The devices of Figure S5b-e were fabricated through the same methods of the main text with different electrode materials and stacking orders.

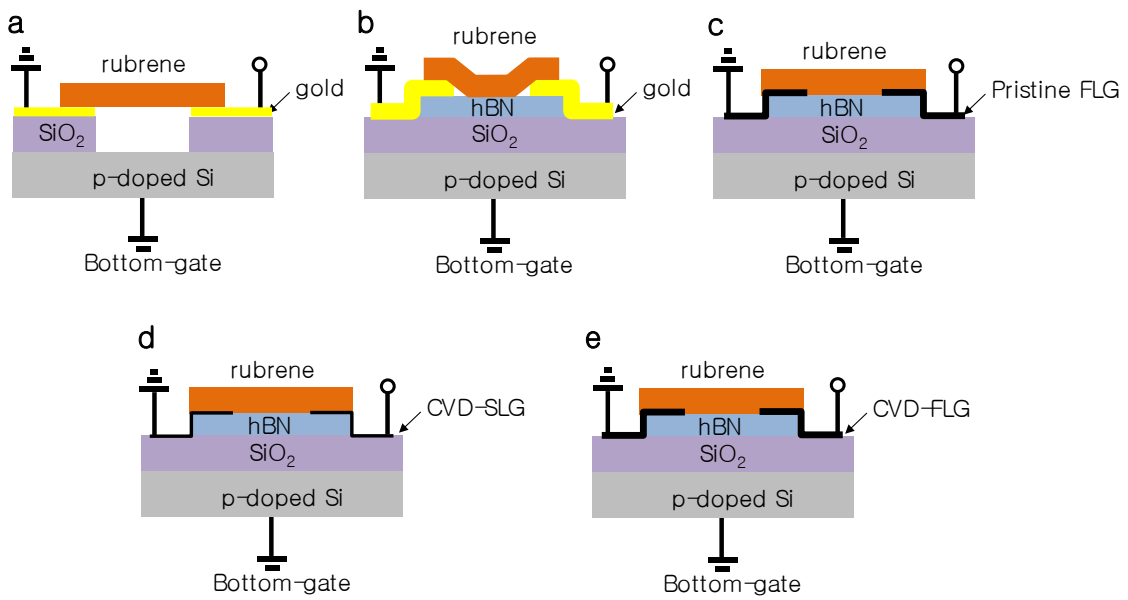

Figure S5. Schematic circuit diagrams of (a) BG-BC with air-gap dielectric and gold electrode, (b) BG-BC with hBN dielectric and gold electrode, (c) BG-BC with hBN dielectric and pristine FLG electrode, (d) BG-BC with hBN dielectric and CVD-SLG electrode, and (e) BG-BC with hBN dielectric and CVD-FLG electrode. 


\section{WILEY-VCH}

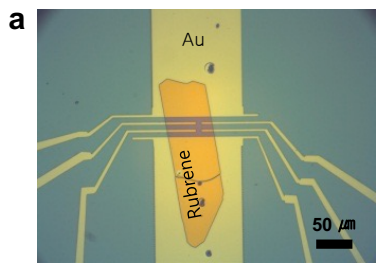

d

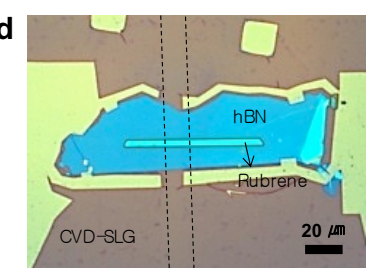

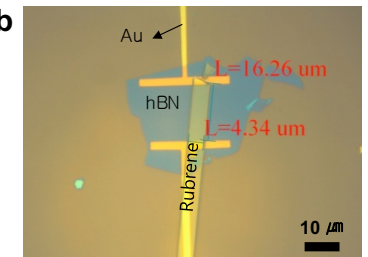
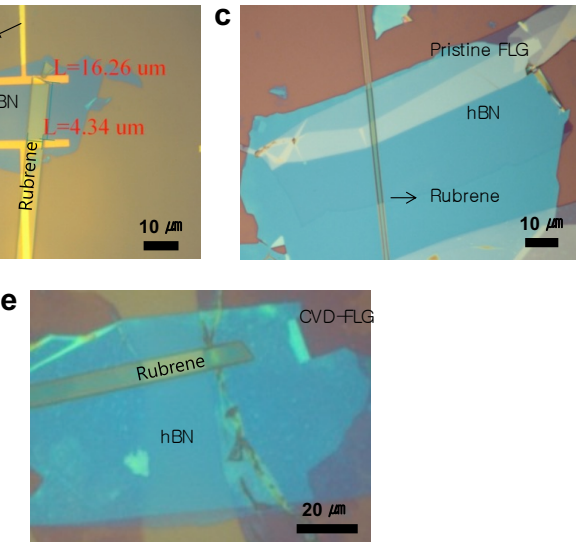

Figure S6. Optical micrographs of (a) BG-BC with air-gap dielectric and gold electrode, (b) BG-BC with hBN dielectric and gold electrode, (c) BG-BC with hBN dielectric and pristine FLG electrode, (d) BG-BC with hBN dielectric and CVD-SLG electrode, and (e) BG-BC with hBN dielectric and CVD-FLG electrode. 
WILEY-VCH

Supporting Table S1

\begin{tabular}{|c|c|c|c|c|c|}
\hline Device & $\begin{array}{c}\text { Device } \\
\text { Structure }\end{array}$ & Dielectric & S/D Electrode & Contact & $\begin{array}{l}\text { Field Effect Mobility } \\
\left(\mathrm{cm}^{2} / \mathrm{Vs}\right)\end{array}$ \\
\hline Ref. 16 & \multirow{6}{*}{ BG-BC } & $\mathrm{SiO}_{2}$ & CVD-SLG & Good & 5.4 \\
\hline Fig. S5a & & Air & $\mathrm{Au}$ & Good & $13-20$ \\
\hline Fig. S5b & & \multirow{4}{*}{ hBN } & $\mathrm{Au}$ & $\mathrm{Bad}$ & 3.8 \\
\hline Fig. S5c & & & Pristine FLG & $\mathrm{Bad}$ & 3.5 \\
\hline Fig. S5d & & & CVD-SLG & Good & 10 \\
\hline Fig. S5e & & & CVD-FLG & $\mathrm{Bad}$ & 2.8 \\
\hline Fig. $4 \mathrm{a}$ & BG-TC & $\mathrm{SiO}_{2}$ & CVD-SLG & $\mathrm{Bad}$ & $<1$ \\
\hline Fig. $4 \mathrm{c}$ & TG-TC & $\mathrm{hBN}$ & CVD-SLG & Good & $10-15$ \\
\hline
\end{tabular}

Table S1. Device performance table of rubrene single crystal based OFET structures with different dielectric and S/D electrodes materials.

The performances of the fabricated devices were summarized in Table S1. The device structures of OFETs can be categorized into bottom-gate bottom-contact (BG-BC), bottomgate top-contact (BG-TC), top-gate bottom-contact (TG-BC), and top-gate top-contact (TGTC). As mentioned in the main text, the TG-TC device with graphene electrode and hBN dielectric showed high mobility comparable to that of the suspended rubrene device. Note that the stacked device in the main text has lower operating voltage. Each contribution of hBN dielectric and graphene electrodes can be found out from mobility enhancement $\left(0.7 \mathrm{~cm}^{2} / \mathrm{Vs}\right.$ $\rightarrow 5.4 \mathrm{~cm}^{2} / \mathrm{Vs}$ by using graphene electrodes, $5.4 \mathrm{~cm}^{2} / \mathrm{Vs} \rightarrow 12.5 \mathrm{~cm}^{2} / \mathrm{Vs}$ by using both graphene electrodes and $\mathrm{hBN}$ dielectric). 